\title{
Does Obesity and Procedure Type Increase the Risk of In-Hospital Mortality in Laparoscopic Hysterectomy: A Report From the United States Hospitals
}

Chris A. Robert ${ }^{1}$, Mary P. Robert ${ }^{2}$, Rikinkumar S. Patel ${ }^{3}$

1. Obstetrics \& Gynecology, Sunrise Hospital, Pune, IND 2. Obstetrics \& Gynecology, LLH Hospital, Abu Dhabi, ARE 3. Psychiatry, Griffin Memorial Hospital, Norman, USA

Corresponding author: Rikinkumar S. Patel, rpatel_09@arcadia.edu

\section{Abstract \\ Objectives}

To assess the differences in demographics and laparoscopic hysterectomy type by comorbid obesity and to assess the risk of in-hospital mortality due to obesity and other comorbidities.

\section{Methods}

We conducted a cross-sectional study using the Nationwide Inpatient Sample (NIS, 2012-2014), and included 119,890 adult females undergoing total laparoscopic hysterectomy (TLH), laparoscopic-assisted vaginal hysterectomy (LAVH), and laparoscopic supracervical hysterectomy (LSH). We used a logistic regression model adjusted for confounders to assess the odds ratio (OR) of obesity on mortality in study inpatients.

\section{Results}

The majority of the inpatients were middle-age 36-50 years (83.1\%) and White (67.7\%). Comorbidities were seen in a higher proportion of obesity cohort with most prevalent being hypertension (53.6\%) and diabetes (23.9\%), followed by depression and hypothyroidism (15.8\% and $15.4 \%$, respectively). Inpatients with comorbid obesity had 4.6 times (95\% CI 2.79-7.69) higher odds for in-hospital mortality compared to nonobesity cohort. There was statistically no significant association between type of laparoscopic hysterectomy and in-hospital mortality.

\section{Conclusion}

Analysis of national-level data shows that obese patients have a higher risk of in-hospital mortality by $364 \%$ compared to non-obese patients. There was no significant association between the laparoscopy procedure type and in-hospital mortality. More studies should focus on improving hospital outcomes and quality of life post-surgery in obese patients.

Received 07/07/2020

Review began $07 / 10 / 2020$ Review ended 07/13/2020 Published 07/22/2020

\section{() Copyright 2020}

Robert et al. This is an open access article distributed under the terms of the Creative Commons Attribution License CC-BY 4.0., which permits unrestricted use, distribution, and reproduction in any medium, provided the original author and source are credited.
Categories: Obstetrics/Gynecology, Quality Improvement

Keywords: laparoscopic assisted vaginal hysterectomy, laparoscopic hysterectomy, comorbid obesity, nationwide inpatient sample, in-hospital mortality, total laparoscopic hysterectomy

\section{Introduction}

Hysterectomies are the second most commonly performed procedure in women of reproductive age, and nearly 600,000 hysterectomies are performed annually. About 20 million women in the United States (US) have had a hysterectomy, and $52 \%$ of all hysterectomies were performed in women under 44 years of age [1]. Laparoscopic hysterectomy was majorly performed for benign indications (83.2\%), with total laparoscopic hysterectomy (TLH) accounting for 48.3\% followed by laparoscopic-assisted vaginal hysterectomy (LAVH, 37.3\%), and laparoscopic supracervical hysterectomy (LSH, 14.4\%) [2]. TLH is the preferred mode of hysterectomy as it affords various advantages, including low postoperative pain, less intraoperative and perioperative blood loss, shorter hospital stay, short convalescence period, and reduced infection rates compared with conventional abdominal hysterectomy [3-5].

According to the Centers for Disease Control and Prevention (CDC), the prevalence of obesity increased from $30.5 \%$ to $42.4 \%$ from the years $1999-2000$ through $2017-2018$. The prevalence was highest among middleaged women (44.8\%) and middle- and lowest-income groups. The medical cost of obesity has been estimated at $\$ 147$ billion in the United States, with the average medical cost $\$ 1,429$ higher in obese [6].

Obesity is often associated with comorbidities like hypertension, diabetes mellitus, and coronary disease and studies show a higher incidence of perioperative deep vein thrombosis, pulmonary embolism, and 
surgical site infection $[7,8]$. However, studies examining the role of obesity in laparoscopic gynecological surgeries have discrepant results with some showing increased risk of complications and conversions to laparotomy, and others showing no difference in between the different weight groups $[9,10]$.

In our study, we aim to assess the differences in demographics and laparoscopic hysterectomy type by the presence of comorbid obesity, and next is to assess the risk of in-hospital mortality due to obesity and other comorbidities.

\section{Materials And Methods \\ Data source}

We conducted a cross-section data analysis using the healthcare cost and utilization project's (HCUP) Nationwide Inpatient Sample (NIS) data from 2012 to 2014. The NIS provides patient records from about 4,400 non-federal hospitals and covers 45 states in the US. Diagnostic and procedure information in the NIS is identified using the International Classification of Diseases, ninth edition (ICD-9) codes, and Clinical Classification Software (CCS) codes [11].

\section{Inclusion criteria and outcome variables}

We included adult patients (age 18 to 50 years) with a primary procedure of laparoscopic hysterectomy, that is, TLH (ICD-9 codes: 68.41 or 68.61), LAVH (ICD-9 codes 68.51 or 68.71), and LSH (ICD-9 code: 68.31). This sample was further grouped by comorbid discharge diagnosis of obesity using the ICD-9 codes 278.0, 278.00, 278.01, 278.03, 649.10-649.14, 793.91, V85.30-V85.39, V85.41-V85.45 or V85.54.

Demographic variables studied included age (18-35 and 36-50 years), and race (white, black, Hispanic, and Asian/Pacific Islanders, Native American, and others). The comorbid diagnosis for depression, diabetes, hypertension, and hypothyroidism were identified using ICD-9 diagnosis codes. We measured the in-hospital mortality between obesity and non-obesity cohorts and in the NIS, we in-hospital mortality is reported as all-cause [12].

\section{Statistical analysis}

We used cross-tabulation and descriptive statistics to discern the demographic and comorbidities differences in the sample population undergoing laparoscopic hysterectomy by comorbid obesity. We used another model of cross-tabulation and descriptive statistics to evaluate the demographic, comorbidities, and hysterectomy type in the sample population by in-hospital mortality. Logistic regression analysis was used to evaluate the demographic and comorbidities that increase the risk of association with in-hospital mortality. A P-value of less than 0.01 was used to determine the statistical significance in all analyses and was conducted using the Statistical Package for the Social Sciences (SPSS), version 26 (IBM Corporation, Armonk, NY).

\section{Ethical approval}

Individual identifiers were used to protect the patient's identity and health-related information. So, the use of de-identified NIS database in this study does not require approval from the institutional review board [11].

\section{Results}

We analyzed a total sample of 119,890 inpatients hospitalized for laparoscopic hysterectomy with 17,370 (14.5\%) inpatients having comorbid obesity. The majority of the inpatients underwent TLH (51.3\%), followed by LAVH (25.8\%) and LSH (12.9\%).

The majority of the inpatients were middle-age adults 36 to 50 years $(83.1 \%)$ with obesity cohort older than non-obesity (51.3y vs. $48.8 \mathrm{y}, \mathrm{P}<0.001$ ). About $67.7 \%$ inpatients were White, and when compared to nonobesity, a higher proportion of obese inpatients were black (17.2\% vs. $12.7 \%)$. Comorbidities were seen in a higher proportion of obesity cohort with most prevalent being hypertension (53.6\%) and diabetes (23.9\%), followed by depression and hypothyroidism (15.8\% and $15.4 \%$, respectively) as shown in Table 1 . 


\section{Cureus}

\begin{tabular}{|c|c|c|c|}
\hline \multirow{2}{*}{ Variable } & \multicolumn{3}{|c|}{ Laparoscopic hysterectomy } \\
\hline & Obesity (-) & Obesity (+) & Total \\
\hline Total inpatients & 102,520 & 17,370 & 119,890 \\
\hline Mean age, years (SD) & $48.8(12.37)$ & $51.3(12.42)$ & - \\
\hline \multicolumn{4}{|l|}{ Age at admission, in \% } \\
\hline $18-35$ years & 17.0 & 16.2 & 16.9 \\
\hline $36-50$ years & 83.0 & 83.8 & 83.1 \\
\hline \multicolumn{4}{|l|}{ Race, in \% } \\
\hline White & 69.2 & 67.7 & 68.9 \\
\hline Black & 12.7 & 17.2 & 13.5 \\
\hline Hispanic & 11.1 & 9.7 & 10.9 \\
\hline Asian/Pacific Islander & 2.4 & 1.1 & 2.2 \\
\hline Native American & 0.6 & 0.9 & 0.6 \\
\hline Others & 4.0 & 3.4 & 3.9 \\
\hline \multicolumn{4}{|l|}{ Comorbidities, in \% } \\
\hline Depression & 9.5 & 15.8 & 10.6 \\
\hline Diabetes & 7.6 & 23.9 & 10.4 \\
\hline Hypertension & 24.9 & 53.6 & 29.9 \\
\hline Hypothyroidism & 10.0 & 15.4 & 11.0 \\
\hline \multicolumn{4}{|l|}{ Hysterectomy type, in \% } \\
\hline LAVH & 37.3 & 28.6 & 35.8 \\
\hline LSH & 13.7 & 8.9 & 12.9 \\
\hline TLH & 48.9 & 62.5 & 51.3 \\
\hline In-hospital mortality, in \% & 0 & 0.1 & 0 \\
\hline
\end{tabular}

TABLE 1: Demographics and hospital outcomes by comorbid obesity in adult inpatients with laparoscopic hysterectomy

SD: standard deviation; TLH: total laparoscopic hysterectomy; LAVH: laparoscopic-assisted vaginal hysterectomy; LSH: laparoscopic supracervical hysterectomy

When divided by type of hysterectomy, the indications for each type in obese patients varied. The majority of the hysterectomies for TLH were performed for cancer of uterus/cervix/ovary (42\%), followed by benign uterine tumors (20\%) and menstrual disorders (15\%). For LSH, benign uterine tumors were the most common indication accounting for $43 \%$ followed by menstrual disorders (25\%) and prolapse of female genital organs (14\%). LAVH was most commonly performed for menstrual disorders (24\%) followed by cancer of uterus/cervix/ovary (22\%) and benign uterine tumors (22\%) as shown in Figure 1. 


\section{Cureus}

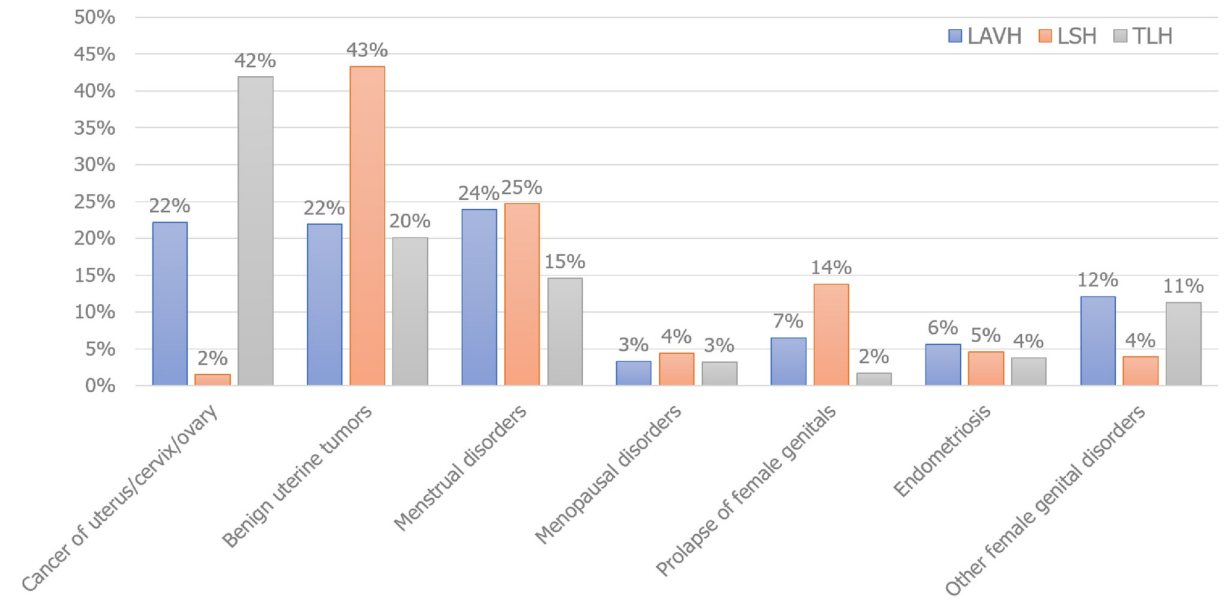

FIGURE 1: Indications in obese women by hysterectomy type

TLH: total laparoscopic hysterectomy; LAVH: laparoscopic-assisted vaginal hysterectomy; LSH: laparoscopic supracervical hysterectomy

Asian/Pacific Islanders and hispanic were at 6.5 times (95\% CI 2.51-16.77) and 2.6 times (95\% CI 1.29-5.21), respectively, higher risk for in-hospital mortality compared to white. Inpatients with comorbid obesity had 4.6 times (95\% CI 2.79-7.69) higher odds for in-hospital mortality compared to non-obesity cohort after controlling for demographic, procedure type, and other comorbidities. There was statistically no significant association between type of laparoscopic hysterectomy and in-hospital mortality as shown in Table 2 . 


\section{Cureus}

\begin{tabular}{|c|c|c|c|c|}
\hline \multirow{3}{*}{ Variable } & \multicolumn{4}{|c|}{ Logistic regression model } \\
\hline & \multirow{2}{*}{ Odds ratio } & \multicolumn{2}{|c|}{$95 \%$ confidence interval } & \multirow{2}{*}{$P$ value } \\
\hline & & Lower & Upper & \\
\hline Age at admission & 1.12 & 1.10 & 1.15 & $<0.001$ \\
\hline \multicolumn{5}{|l|}{ Race } \\
\hline White & \multicolumn{4}{|l|}{ Reference } \\
\hline Black & 2.28 & 1.12 & 4.65 & 0.023 \\
\hline Hispanic & 2.59 & 1.29 & 5.21 & 0.008 \\
\hline Asian/Pacific Islander & 6.49 & 2.51 & 16.77 & $<0.001$ \\
\hline Native American & $<0.001$ & $<0.001$ & - & 0.990 \\
\hline Others & $<0.001$ & $<0.001$ & - & 0.996 \\
\hline \multicolumn{5}{|l|}{ Comorbidities } \\
\hline No comorbidity & \multicolumn{4}{|l|}{ Reference } \\
\hline Depression & 4.14 & 2.41 & 7.09 & $<0.001$ \\
\hline Diabetes & 1.26 & 0.73 & 2.19 & 0.411 \\
\hline Hypertension & 0.54 & 0.31 & 0.93 & 0.027 \\
\hline Hypothyroidism & 1.11 & 0.62 & 1.99 & 0.737 \\
\hline Obesity & 4.64 & 2.79 & 7.69 & $<0.001$ \\
\hline \multicolumn{5}{|l|}{ Hysterectomy type } \\
\hline LAVH & 1.59 & 0.96 & 2.66 & 0.073 \\
\hline LSH & 1.11 & 0.43 & 2.86 & 0.837 \\
\hline TLH & Reference & & & \\
\hline
\end{tabular}

\section{TABLE 2: In-hospital mortality risk in adult inpatients with laparoscopic hysterectomy}

TLH: total laparoscopic hysterectomy; LAVH: laparoscopic-assisted vaginal hysterectomy; LSH: laparoscopic supracervical hysterectomy

By proportion, $0.1 \%$ (35 out of 17,370) in-hospital mortality was seen in obesity cohort. A higher number of inpatient deaths were reported in patients who underwent TLH $(N=25)$ and LAVH $(N=10)$, and none in those undergoing LSH. A total of 45 inpatient deaths were reported in 102,520 inpatients with 20 deaths each in inpatients undergoing TLH and LAVH as shown in Figure 2. 


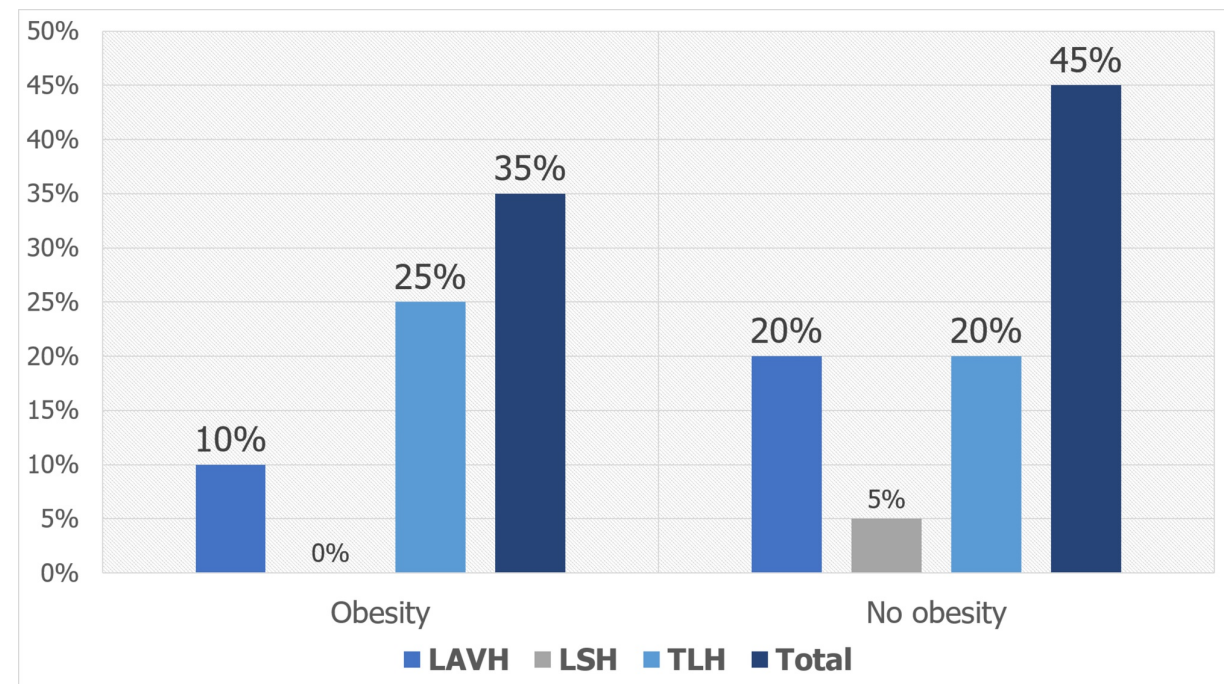

FIGURE 2: In-hospital mortality by hysterectomy type in adult inpatients

TLH: total laparoscopic hysterectomy; LAVH: laparoscopic-assisted vaginal hysterectomy; LSH: laparoscopic supracervical hysterectomy

\section{Discussion}

In this study, utilizing the NIS data analysis of 119,890 inpatients hospitalized for primary procedure: laparoscopic hysterectomy, we found that 17,370 (14.5\%) had comorbid obesity. When the obese and the non-obese cohorts were compared, we found that there was a higher proportion of comorbidities in the obese cohort with hypertension being the most prevalent, followed by diabetes, depression, and hypothyroidism. Patients with comorbid obesity had a 364\% higher risk of in-hospital mortality compared to the non-obese cohort.

More than four-fifth of the inpatients were middle-aged adults (36 to 50 years, $83.1 \%$ ), with the obesity cohort older than non-obesity. The CDC health trend data also reveals that the prevalence of obesity was highest among middle-aged women and the black population [6]. In our research, $67.7 \%$ of total inpatients were white, but when compared to non-obesity, a higher proportion of obese inpatients were black. We found that the Asian/Pacific Islanders and Hispanic populations were at 6.5 times and 2.6 times, respectively, higher risk of in-hospital mortality compared to the White population. This may be because racial minority groups are disproportionately affected by chronic diseases compared to their white counterparts due to lower socioeconomic status and education level, as well as a lack of accessibility to health care and nutritious food. These factors can hinder such groups from receiving adequate medical care and nutrition, leading to an increased incidence of obesity and the risk of complications following surgical procedures [13].

Obesity was deemed to be a relative contraindication for laparoscopy as they are at a higher risk of developing atelectasis as a consequence of the upward displacement of the diaphragm following carbon dioxide insufflation. The loss of muscle tone due to anesthesia and the Trendelenburg position leads to a more definite reduction in functional residual capacity in obese [14-17]. In addition, its association with other comorbid conditions can also negatively impact the outcomes of surgical patients $[13,18,19]$. In this study, we found that the most common comorbidities were hypertension (53.6\%) and diabetes (23.9\%). We found a significant association between comorbid obesity and in-hospital mortality in the inpatients for laparoscopic hysterectomy after controlling for demographics and chronic comorbidities. Also, the largeness of the abdominal wall results in difficult access to and visualization of the abdominal cavity, pneumoperitoneum maintenance, and laparoscopic instrument handling [20].

Studies exploring the complication rates in obese and non-obese patients have produced conflicting results. Some have found increased complications and laparo-conversions in obese patients, while other studies suggest that there are no relevant differences between the weight groups $[9,10,21]$. In a review of 2,530 laparoscopic hysterectomies, those with a BMI higher than 30 kilograms/square meter had a two-fold risk of unintended laparotomy $[20,22]$. A study by O'Hanlan et al. reported comparable mean operating time, mean operative blood loss, mean length of hospital stay, and complication rates in all BMI groups [18].

While these studies compare the complication rates in the obese and non-obese groups, there is a dearth of literature on the differences in inpatient mortality in at-risk patients undergoing laparoscopic hysterectomy. Therefore, our goal was to examine this outcome comparison because, with the increasing prevalence of obesity, it is of the utmost importance to study, introspect, and implement practices to give a safe outcome 
to patients. In our study, patients with comorbid obesity had 4.6 times and about $364 \%$ higher risk of inhospital mortality compared to the non-obesity cohort. This was in accordance with a prospective, multicenter study of 118,707 patients who underwent non-bariatric general surgery where higher mortality risk was observed in underweight and morbidly obese [23].

Of the $0.1 \%$ (35 out of 17,370 ) of the in-hospital mortality that was seen in the obesity cohort in our study, a higher number of inpatient deaths were reported in patients who underwent TLH and LAVH, while none were reported in those undergoing LSH. There was no statistically significant association between the type of laparoscopic hysterectomy and in-hospital mortality. Milad et al. reported different findings that showed LAVH to be associated with higher morbidity than LSH [24].

The findings of this study must be seen in the light of some limitations. Firstly, the NIS is an administrative database and not a detailed medical and patient record. We categorized laparoscopic hysterectomy based on ICD-9 procedure codes which are subject to possible miscoding and unable to get details of surgical techniques used. Also, NIS data be deficient in preoperative information: prior abdominal surgeries, size, and descent of the uterus. Next, as ICD-9 diagnostic codes were used to identify comorbid obesity and so it could not be stratified into different classes. Studies have shown that an "obesity paradox" exists, where moderate obesity is protective and mortality is most likely in a morbidly obese individual [25]. However, this phenomenon could not be tested in our study.

\section{Conclusions}

Analysis of national-level data shows that obese patients have a higher risk of in-hospital mortality by $364 \%$ compared to non-obese patients. Women with obesity had a higher proportion of chronic comorbidities including hypertension, and diabetes. There was no significant association between the laparoscopy procedure type and in-hospital mortality, yet we found that a higher proportion of inpatient deaths were reported in those who underwent TLH and LAVH. More studies should focus on improving hospital outcomes and quality of life post-laparoscopy in obese women.

\section{Additional Information \\ Disclosures}

Human subjects: Consent was obtained by all participants in this study. Animal subjects: All authors have confirmed that this study did not involve animal subjects or tissue. Conflicts of interest: In compliance with the ICMJE uniform disclosure form, all authors declare the following: Payment/services info: All authors have declared that no financial support was received from any organization for the submitted work. Financial relationships: All authors have declared that they have no financial relationships at present or within the previous three years with any organizations that might have an interest in the submitted work. Other relationships: All authors have declared that there are no other relationships or activities that could appear to have influenced the submitted work.

\section{References}

1. Hysterectomy surveillance --- united states, 1994--1999 . (2002). Accessed: March 23, 2020: https://www.cdc.gov/mmwr/preview/mmwrhtml/ss5105a1.htm.

2. Desai VB, Guo XM, Fan L, Wright JD, Xu X: Inpatient laparoscopic hysterectomy in the United States: trends and factors associated with approach selection. J Minim Invasive Gynecol. 2017, 24:151-158. 10.1016/j.jmig.2016.08.830

3. Olsson JH, Ellstrom M, Hahlin M: A randomised prospective trial comparing laparoscopic and abdominal hysterectomy. Br J Obstet Gynaecol. 1996, 103:345-350. 10.1111/j.1471-0528.1996.tb09740.x

4. Janda M, Gebski V, Brand A, et al.: Quality of life after total laparoscopic hysterectomy versus total abdominal hysterectomy for stage I endometrial cancer (LACE): a randomised trial. Lancet Oncol. 2010, 11:772-780. 10.1016/S1470-2045(10)70145-5

5. Pellegrino A, Signorelli M, Fruscio R, et al.: Feasibility and morbidity of total laparoscopic radical hysterectomy with or without pelvic limphadenectomy in obese women with stage I endometrial cancer. Arch Gynecol Obstet. 2009, 279:655-660. 10.1007/s00404-008-0790-5

6. Adult obesity facts. (2020). Accessed: March 23, 2020: https://www.cdc.gov/obesity/data/adult.html.

7. DeMaria EJ, Carmody BJ: Perioperative management of special populations: obesity. Surg Clin North Am. 2005, 85:1283-1289. 10.1016/j.suc.2005.09.002

8. Anaya DA, Dellinger EP: The obese surgical patient: a susceptible host for infection . Surg Infect. 2006, 7:473-480. 10.1089/sur.2006.7.473

9. Bijen $\mathrm{CB}$, de Bock GH, Vermeulen KM, et al.: Laparoscopic hysterectomy is preferred over laparotomy in early endometrial cancer patients, however not cost effective in the very obese. Eur J Cancer. 2011, 47:21582165. 10.1016/j.ejca.2011.04.035

10. Mueller A, Thiel F, Lermann J, Oppelt P, Beckmann MW, Renner SP: Feasibility and safety of total laparoscopic hysterectomy (TLH) using the Hohl instrument in nonobese and obese women. J Obstet Gynaecol Res. 2010, 36:159-164. 10.1111/j.1447-0756.2009.01112.x

11. Overview of the national (nationwide) inpatient sample . (2019). Accessed: March 15, 2020: https://www.hcup-us.ahrq.gov/nisoverview.jsp.

12. NIS description of data elements . (2019). Accessed: March 15, 2020: https://www.hcupus.ahrq.gov/db/nation/nis/nisdde.jsp. 
13. Choban PS, Flancbaum L: The impact of obesity on surgical outcomes: a review . J Am Coll Surg. 1997, 185:593-603. 10.1016/s1072-7515(97)00109-9

14. Camanni M, Bonino L, Delpiano EM, Migliaretti G, Berchialla P, Deltetto F: Laparoscopy and body mass index: feasibility and outcome in obese patients treated for gynecologic diseases. J Minim Invasive Gynecol. 2010, 17:576-582. 10.1016/j.jmig.2010.04.002

15. Meininger D, Zwissler B, Byhahn C, Probst M, Westphal K, Bremerich DH: Impact of overweight and pneumoperitoneum on hemodynamics and oxygenation during prolonged laparoscopic surgery. World J Surg. 2006, 30:520-526. 10.1007/s00268-005-0133-7

16. Tokics L, Hedenstierna G, Strandberg A, Brismar B, Lundquist H: Lung collapse and gas exchange during general anesthesia: effects of spontaneous breathing, muscle paralysis, and positive end-expiratory pressure. Anesthesiology. 1987, 66:157-167.

17. Safran DB, Orlando R 3rd: Physiologic effects of pneumoperitoneum. Am J Surg. 1994, 167:281-286. 10.1016/0002-9610(94)90094-9

18. O'Hanlan KA, Lopez L, Dibble SL, Garnier AC, Huang GS, Leuchtenberger M: Total laparoscopic hysterectomy: body mass index and outcomes. Obstet Gynecol. 2003, 102:1384-1392. 10.1016/j.obstetgynecol.2003.08.018

19. GBD 2015 Obesity Collaborators, Afshin A, Forouzanfar MH, et al.: Health effects of overweight and obesity in 195 countries over 25 years. N Engl J Med. 2017, 377:13-27. 10.1056/NEJMoa1614362

20. Eltabbakh GH, Piver MS, Hempling RE, Recio FO: Laparoscopic surgery in obese women. Obstet Gynecol. 1999, 94:704-708. 10.1016/s0029-7844(99)00406-8

21. McMahon MD, Scott DM, Saks E, Tower A, Raker CA, Matteson KA: Impact of obesity on outcomes of hysterectomy. J Minim Invasive Gynecol. 2014, 21:259-265. 10.1016/j.jmig.2013.08.707

22. Sokol AI, Chuang K, Milad MP: Risk factors for conversion to laparotomy during gynecologic laparoscopy . J Am Assoc Gynecol Laparosc. 2003, 10:469-473. 10.1016/s1074-3804(05)60146-6

23. Mullen JT, Moorman DW, Davenport DL: The obesity paradox: body mass index and outcomes in patients undergoing nonbariatric general surgery. Ann Surg. 2009, 250:166-172. 10.1097/SLA.0b013e3181ad8935

24. Milad MP, Morrison K, Sokol A, Miller D, Kirkpatrick L: A comparison of laparoscopic supracervical hysterectomy vs laparoscopically assisted vaginal hysterectomy. Surg Endosc. 2001, 15:286-288. $10.1007 /$ s004640000328

25. Amundson DE, Djurkovic S, Matwiyoff GN: The obesity paradox. Crit Care Clin. 2010, 26:583-596. 10.1016/j.ccc.2010.06.004 